\title{
A current-based SCOPF with enhanced particle swarm optimization
}

\author{
Cong-Hui Huang ${ }^{\mathrm{a} *}$, Yih-Feng Su ${ }^{\mathrm{a}}$, Bo-Ren Shih ${ }^{\mathrm{a}}$, Chao-Chin Chiang ${ }^{\mathrm{b}}$, \\ Jheng-Han Lin ${ }^{\mathrm{b}}$, and Cang-Song $\mathrm{Syu}^{\mathrm{b}}$ \\ ${ }^{a}$ Far East University/Automation and Control Engineering, Tainan City 74448, Taiwan \\ ${ }^{b}$ Far East University/ Mechanical Engineering, Tainan City 74448, Taiwan
}

\begin{abstract}
A current-based security-constrained optimal power flow (SCOPF) is presented in this paper and an Enhanced Particle Swarm Pptimization (EPSO) is developed to solve the non-convex optimal power flow problem. The SCOPF can be divided into three steps involving security analysis, severest event selection, and a preventive algorithm. Firstly, novel security analysis is conducted before a fault occurs in the system using the current-based power flow technique. Secondly, a ranking method is used to highlight the most severe events caused by a specific facility. Finally, a preventive algorithm makes use of the contingency information, and can maintain the operator system security, avoiding congestion when a fault occurs. In addition, this method not only enhances the neighborhood search, but also searches for the optimum solution quickly to advance the convergence.
\end{abstract}

Keywords: Optimal power flow, particle swarm optimization, security analysis, security-constrained optimal power flow.

\section{Introduction}

Since the Optimal Power Flow (OPF) was discussed by Carpentier [1] in 1962, many authors have researched solutions to the OPF problem. Many algorithms were developed including methods of directed optimization of the non-linear OPF problem [2],[3]. OPF can be applied not only in system planning, but also in real-time operation for power systems in a deregulated environment. The general solution of the OPF consists of minimizing an objection function subject to equal and unequal constraints. If the constraints are not only considered under normal operation but also under contingencies, the OPF becomes a Security-Constrained Optimal Power Flow (SCOPF).

In recent years, power system loading has continued to increase in all countries. It is difficult to add power plants and power transmission lines, which causes power dispatch problems. Numerous accidents of voltage collapse have taken place. Therefore, power companies and researchers are paying more attention to power system security issues. The main reasons for the occurrence of these events are: 1) bus load continues to expand (direct factor), and 2) the disturbance caused by overloaded power lines or overrange electrical energy of the generators in this system (indirect factor) [4]. In general, the central dispatch unit of the Power Company is responsible for monitoring and dispatching the operating power system. The duty dispatchers determine the operating situation of the system from the central dispatch unit, which the measured parameters have been sent to by a specific communication system in all loading areas. The function of the central dispatch unit is primarily concerned with system security and can be divided into three parts: i) system monitoring, ii) security analysis (SA) or contingency analysis, and iii) security-constrained optimal power flow [5].

\footnotetext{
* Manuscript received June 24, 2013; revised October 4, 2013.

Corresponding author: Cong-Hui Huang, Tel.: +886-6-5979566ext.5527; E-mail address: ch_huang@cc.feu.edu.tw.
} 
The security mechanism of the electric power dispatching unit design can be divided into preceding and past failures of the security dispatch [5]. The security dispatcher of a preceding failure is the optimized power flow program which operates under an ideal situation which is limited by the system data and data execution safety. The function of the program is to control all adjustable values and avoid accidents when the system is operated at violating limits. SCOPF can consider occasional accidents and calculate the adjustable values, such as generator power and voltage, transformer tap, electricity trading, etc. The security dispatch of a past failure is based on SCOPF results, when a failure occurs, power dispatchers can dispatch for the system according to their experience.

In industry, the OPF can only deal with continuous variables. If we wish to join the objective function or restricted equation into the security restrictions, OPF cannot currently solve related issues [6]. Artificial Intelligence techniques have been developed in recent years, including, genetic algorithms (GA), evolutionary programming (EP), ant colony optimization (ACO), particle swarm optimization (PSO), etc. [6]-[10]. In view of this, we propose the hybrid genetic-ant colony algorithm (HGACA) to solve the security restrictions of optimized power flow. The technique is based on a power flow model for the current-based method [11], [12], and combined with an interior point algorithm to solve for the continuous variables of OPF. For discrete variables under security restrictions, we adopt HGACA to solve for them, and propose preventive CSOPF for preventative security restrictions. The paper proposes a new operation method, CSOPF, to prevent contingences from resulting in serious damage. CSOPF can reduce the contingency risk and lead to a power system that is secure and economic to operate.

\section{Technical Background}

\subsection{Enhanced particle swarm optimization}

$\mathrm{PSO}$, as a population-based algorithm, exploits a population of individuals to probe promising regions of the search space. The population is called a swarm and the individuals are the particles. The basic PSO algorithm is shown in Fig. 1.

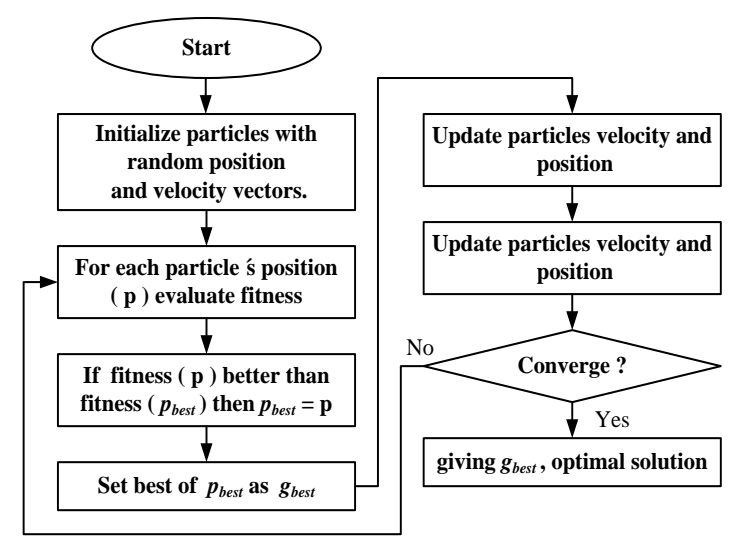

Fig. 1. Basic PSO algorithm.

As the swarm iterates, the fitness of the global best solution improves (decreases for a minimization problem). It is expected that all particles being influenced by the global best solution eventually approach the global best solution. If the fitness does not improve despite the PSO iterations increasing, the convergence is said to have been reached. In the pioneering work of Kennedy and Eberhart in 1995 [10], the particle position and velocity was defined as

$$
\begin{aligned}
V_{[I][J]}^{k+1} & =V_{[I][J]}^{k}+C_{1} \operatorname{rand}_{1}\left(X_{-} \text {Lbest }_{[I][J]}^{k}-X_{[I][J]}^{k}\right)+C_{2} \operatorname{rand}_{2}\left(X_{-} \text {Gbest }_{[I][J]}^{k}-X_{[I][J]}^{k}\right), \\
X_{[I][J]}^{k+1} & =X_{[I][J]}^{k}+V_{[I][J]}^{k+1}
\end{aligned}
$$


where $[I]$ and $[J]$ are the population number and particles number, $V_{[I][J]}^{k}$ is the velocity of the particle in the $k^{\text {th }}$ iteration, $X_{[I][J]}^{k}$ is the position of the particle in the $k^{\text {th }}$ iteration, $X_{-}$Lbest $_{[I][J]}^{k}$ is the $I^{\text {th }}$ fitness best in the $k^{\text {th }}$ iteration, $X_{-}$Gbest $_{[I][J]}^{k}$ is the population global best in the $k^{\text {th }}$ iteration, $C_{1}, C_{2}$ are the cognitive and social component, respectively. These components influence how much the particle's personal best and the global best (respectively) influence its movements, and rand $_{1}$, rand $_{2}$ are uniform random numbers between 0 and 1 .

The EPSO produces a diverse range of positions by recognizing the affinities between position and global best solution, or between positions. Through the diversity embodiment, the quality of solutions in the feasible space is improved. In regards to the diversity, EPSO uses the Euclidean distance (ED) to cover for information entropy in Immune Algorithm (IA) [13]. The diversities of each position are calculated between the $2 \times$ PS positions (the last PS position and the current PS position) and the best position. If we let the best position be $y_{i}(0)$, where $i=1,2,3, \ldots, N$, and the $2 * \mathrm{PS}$ competitive positions be $Y(k)=\left[y_{1}(k), \mathrm{y}_{2}(\mathrm{k}), \ldots, y_{i}(k), \ldots, y_{N}(k)\right]$, where $k=1,2,3, \ldots, 2 \times \mathrm{PS}$, the best position is

$$
Y_{\text {best }}=\left[\begin{array}{llllll}
y_{1}(0) & y_{2}(0) & \cdots & y_{i}(0) & \cdots & y_{N}(0)
\end{array}\right] \text {. }
$$

The Euclidean distance between the best and competitive positions are calculated as

$$
E D(k)=\sqrt{\sum_{i=1}^{N}\left[\left|y_{i}(0)-y_{i}(k)\right|\right]^{2}} .
$$

There are two types of affinity in EPSO. The first is to elucidate the relationships between two positions. Therefore, the mutual diversity of positions can be evaluated as

$$
\left(A f f_{b}\right)^{k}=(1+E D(k))^{-1},
$$

where $\left(A f f_{b}\right)^{k}$ is the affinity between the best position and the k-th position. If all genes within these two positions are the same, $E D(k)$ is zero and the k-th affinity is equal to one. Note that this affinity value is located between zero and one.

The second affinity is between the position and the global best, where the combination intensity between the objective and the solution is investigated as

$$
\left(A f f_{g}\right)^{k}=\mathrm{Obj}_{-} f_{k},
$$

where $O b j_{-} f_{k}$ is a value of the objective function with k-th position.

\subsection{Amending the contingency of the system model}

Generally speaking, the contingencies are classified using an amended generator contingency model and an amended transmission line contingency model, as shown in Fig. 2 and Fig. 3, respectively.

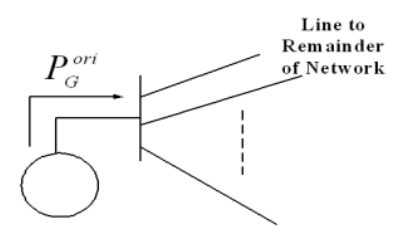

Fig. 2. Amended generator contingency model.

$P_{G}^{F}=P_{G}^{o r i}+\Delta P_{G}$,

$\Delta P_{G}=-P_{G}^{o r i}$, 


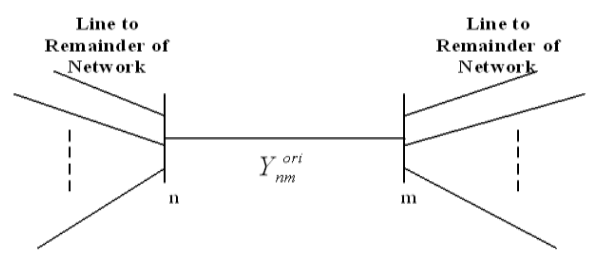

Fig. 3. Amended transmission line contingency model.

where $P_{G}{ }^{F}$ is the power of the generator after contingency, $P_{G}{ }^{\text {ori }}$ is the power of the generator before contingency, and and $\Delta P_{G}$ is the amended power of the generator.

$$
\begin{aligned}
{\left[Y_{\text {matrix }}^{F}\right] } & =\left[Y_{\text {matrix }}^{\text {ori }}\right]+\left[\Delta Y_{n m}\right] \\
{\left[\Delta Y_{n m}\right]=} & {\left[\begin{array}{cccccc}
\ddots & & & & & \cdot \\
\cdots & \Delta Y_{n n}^{\prime} & \cdots & \cdots & \Delta Y_{n m} & \cdots \\
\vdots & \ddots & & \vdots & \\
& \vdots & & \ddots & \vdots & \\
\cdots & \Delta Y_{n m} & \cdots & \cdots & \Delta Y_{m m}^{\prime} & \cdots \\
\cdot & & & & & \ddots
\end{array}\right] }
\end{aligned}
$$

where $\left[Y_{\text {matrix }}^{\text {ori }}\right]$ is the conductivity matrix after contingency, $\left[Y_{\text {matrix }}^{F}\right]$ is the conductivity matrix before contingency, and $\left[\Delta Y_{n m}\right]$ is the amended conductivity matrix.

\subsection{Modified security analysis}

SA is required to analyze all accident sources from potentially dangerous systems in a very short period of time. If we use traditional SA to analyze all generators and transmission lines, it cannot be achieved, while meeting the requirement of a fast solver. In this study, we propose an analysis flow to improve the analysis efficiency, shown in Fig. 4.

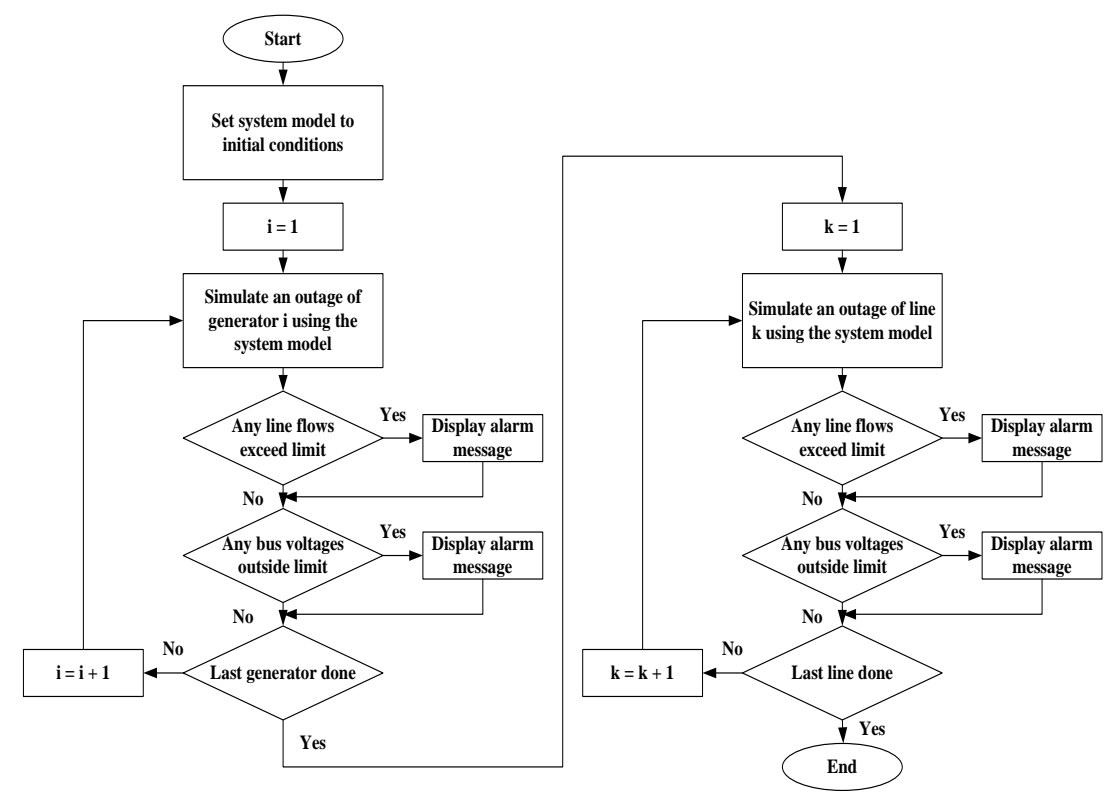

Fig. 4. Flowchart of modified security analysis. 


\section{Preventive SCOPF}

SCOPF combined with optimized power flow can determine the best adjustable location of controllable values from generators under occasional accident analysis. When the security analysis function was executed, the system will not incur any occasional accidents and operate at violating limits. The system operating parameters were modified and adjusted to result in a security dispatch state that avoids violating limits when the system operation is normal. The objective function of the optimized power flow is to reduce the cost of electricity as

$$
\text { Min } f(x)=\sum_{i=1}^{N G} a_{i} P_{G i}^{2}+b_{i} P_{G i}+c_{i},
$$

where the system constraints could be composed of the power balance (11) and inequality constraints (12).

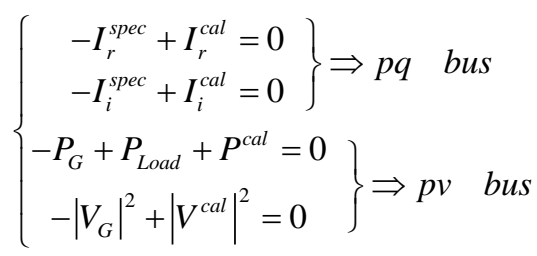

$$
\begin{aligned}
& \left\{\begin{array}{l}
S_{i j}^{2} \leq \bar{S}_{L, i j}^{2} \\
S_{j i}^{2} \leq \bar{S}_{L, i j}^{2} \\
\underline{P_{G i}} \leq P_{G i} \leq \overline{P_{G i}} \\
\left|\underline{V}_{i}\right|^{2} \leq\left(e_{i}^{2}+f_{i}^{2}\right) \leq\left|\overline{V_{i}}\right|^{2}
\end{array}\right.
\end{aligned}
$$

where $a_{i}, b_{i}, c_{i}$ are the fuel cost coefficients of thermal plan $I,\left|V_{i}\right|$ is the voltage magnitude at bus $I, e_{i}, f_{i}$ are the real and imaginary parts of voltage $V_{i}$ at bus $I, P_{G i}$ is dispatchable active power at bus $I, S_{i j}^{2}, S_{j i}^{2}$ are apparent power of transmission line $(i, j)$ or $(j, i)$, and $\bar{S}_{L, i j}^{2}, \bar{S}_{L, j i}^{2}$ are the apparent power limit of transmission line $(i, j)$ or $(j, i)$, where $\bar{S}_{L, i j}^{2}=\bar{S}_{L, j i}^{2}$.

SCOPF take SA into consideration when the most hazardous cases occur. Before a system accident, it will already consider the most dangerous situations in the optimal power flow. If the accident happens for the most dangerous case, the system can safely operates under any constraints without changing any settings. If the accident instead occurs in one of the other cases, the system could recover to safe operation by adjusting some settings, which does not cause mortal influence. The general OPF cannot deal with the original system and accident system together, so we propose EPSO to solve these types of problems in this study. We first need to solve the optimal dispatching by OPF for the original system and join the most dangerous case into EPSO. In this step, we only need to consider the violation of the restricted equation in the most dangerous case under the same optimal dispatching project without solving the objective function. Therefore, the optimal dispatching result of SCOPF is the minimum cost of electricity in the most dangerous case.

\section{Numerical Simulation}

This section presents some numerical results obtained with the implementation of SCOPF in a modified IEEE 30 buses system.

\subsection{System security analysis}

The load flow of the current injection method has the advantage of fast convergence. When performing 
SA, the results can be obtained from direct convergence. For the modified IEEE 30 Bus, 50 load flows are required for the system for each SA performed (5 power generators and 45 power delivery cables). The total time required is merely 0.547 seconds. In the case where SA is to be performed on the IEEE 118 Bus (5 power generators and 186 power delivery cables) and IEEE 300 Bus (68 power generators and 411 power delivery cables), the time required is 3.265 seconds and 12.536 seconds, respectively. Table 1 lists the system damage as a result of performing SA under the 30 Bus model, in the order of severity.

Table 1 . Top 10 cases for system damage

\begin{tabular}{clcc}
\hline ID & Faulty unit & Overdrive power delivery cable & Overdrive flow (MW) \\
\hline 1 & G4 & Line 6-8 & 3.73 \\
2 & Line 8-9 & Line 6-8 & 3.03 \\
3 & Line 10-22 & Line 21-22 & 2.46 \\
4 & Line 4-6 & Line 21-22 & 1.64 \\
5 & G2 & Line 6-8 & 0.81 \\
6 & Line 8-28 & Line 6-8 & 0.41 \\
7 & Line 10-20 & Line 15-18 & 0.40 \\
8 & Line 15-23 & Line 23-24 & 0.39 \\
9 & Line 12-13 & Line 16-17 & 0.29 \\
10 & Line 1-3 & Line 21-22 & 0.08 \\
\hline
\end{tabular}

It can be observed from Table 1, that under such circumstances as a fault at G4, line 6-8 would experience overdrive (surpass $3.73 \mathrm{MW}$ )

\subsection{Optimal dispatch without security analysis}

In this test, OPF was solved under an accident situation that was ignored. This kind of question belongs to continuous nonlinear problems which could be solved by original OPF [12] or an AI method. Irrespective of the algorithm used to solve the problem, we can obtain the same optimal dispatching. The objective function of test is the minimum cost of electricity, the results showed that the power generator cost is $487.4019 \$ / \mathrm{h}$. The optimal dispatching results are shown in Table 2.

Table 2. OPF dispatch without security analysis

\begin{tabular}{|c|c|c|c|c|c|c|}
\hline Items Bus & Gen 1 & Gen 2 & Gen 3 & Gen 4 & Gen 5 & Gen 6 \\
\hline$P_{G}(\mathrm{MW})$ & 19.257 & 76.916 & 21.22 & 38.425 & 30 & 15.576 \\
\hline$\left|V_{G}\right|$ (p.u.) & 1.0038 & 1.0934 & 1.0042 & 1.0358 & 1.0061 & 1.0038 \\
\hline
\end{tabular}

\subsection{Optimal dispatch with SCOPF}

In this test, we develop EPSO as a tool to solve the optimal power flow, and the load flow of the power injection method as a system limit tool. The restricted function and inequality restricted function of the objective function of the test is the minimum cost of electricity (11) and (12). The electrical energy and voltage of the generator are control variables which combined with SA can obtain the most dangerous case (G4 fault). After testing, the optimal dispatching is given in Table 3, where the modified IEEE 30 Bus equation was used as the test system. When the dangerous case, G4, was broken down, the dispatching result of PSCOPF can still operate safely without changes to any settings.

\subsection{SCOPF with G4 fault}

In the case where G4 is at fault and the most system damage occurs, the adjusted results from SCOPF still ensure that the system operates safely without any configuration change. When the power generator G4 (Bus 22) is at fault, this particular bus is changed to the PQ Bus. The power generator on the swing Bus will automatically adjust its output power to obtain balance. All other power generators remain at their original output power and their voltage ratio with the Buses, as shown in Table 3 . At this time, the system satisfies all limitations and is working safely under normal operation. 
Table 3. Optimal dispatch of SCOPF

\begin{tabular}{cccccc}
\hline Gen & $P_{G}(\mathrm{MW})$ & $P_{G}{ }^{+}(\mathrm{MW})$ & $\left|V_{G}\right|$ (p.u.) & $\left|V_{G}\right|^{+}$p.u. $)$ & $\left|V_{G}\right|^{-}$(p.u.) \\
\hline G1 & 20.39 & 100 & 1.050 & 1.10 & 0.90 \\
G2 & 46.30 & 120 & 1.038 & 1.10 & 0.90 \\
G3 & 32.52 & 60 & 1.055 & 1.10 & 0.90 \\
G4 & 32.38 & 50 & 1.039 & 1.10 & 0.90 \\
G5 & 25.67 & 60 & 1.058 & 1.10 & 0.90 \\
G6 & 33.85 & 70 & 1.067 & 1.10 & 0.90 \\
\hline$P_{G}{ }^{+}:$upper limit of generator power; & & & \\
$\mid V_{G}{ }^{+}:$upper limit of generator voltage; & \\
$\left|V_{G}\right|^{-}:$lower limit of generator voltage; &
\end{tabular}

Table 4. System status when G4 is at fault

\begin{tabular}{cccccc}
\hline Gen & $P_{G}(\mathrm{MW})$ & $P_{G}{ }^{+}(\mathrm{MW})$ & $\left|V_{G}\right|$ (p.u.) & $\left|V_{G}\right|^{+}$p.u. $)$ & $\left|V_{G}\right|^{-}$(p.u.) \\
\hline G1 & 54.18 & 100 & 1.050 & 1.10 & 0.90 \\
G2 & 46.30 & 120 & 1.038 & 1.10 & 0.90 \\
G3 & 32.52 & 60 & 1.055 & 1.10 & 0.90 \\
G4 & 0 & & 1.00094 & & 0.90 \\
G5 & 25.67 & 60 & 1.058 & 1.10 & 0.90 \\
G6 & 33.85 & 70 & 1.067 & 1.10 & 0 \\
\hline
\end{tabular}

\section{Conclusion}

In this study, we performed system safely analysis on an integrated accident correction model using load flow based on the current injection method. The test results confirmed that a significant amount of time is saved when compared to the conventional SA method. The advantage of a complete convergence for the power flow helps to obtain the true representation of the actual system status during analysis. In addition, we proposed that the time saved could be used to find the optimal power flow under safety constraints using MPSO. The system can adjust and modify its safety measurement according to the SCOPF results where the most severe cases are identified. In the case where accidents occur, it prevents the system from operating beyond its regular constraints. In addition, additional configuration changes would not be required, and the system can still operate safely under normal constraints. Safety constraints are very complex, and the MPSO method proposed in our study is able to obtain a precise solution for adjustment. In terms of the convergence time and quality, the MPSO method is better than the conventional particle swarm optimization.

\section{Acknowledgements}

We would like to thank the National Science Council of the Republic of China, Taiwan (Contract No. NSC 102-2221-E-269 -018 and NSC 102-3113-P-194 -002).

\section{References}

[1] Carpienter J., Contribution I'etude do dispatching economique. Bulletin Society Francaise Electriciens, 1962, 3(1): $431-447$.

[2] Huneault M, and Galiana F. D., A survey of the optimal power flow literature. IEEE Trans. on Power Systems, 1991, 6(2): 762-770.

[3] Verbic G., and Canizares C. A., Probabilistic optimal power flow in electricity markets based on a two-point estimate method. IEEE Trans. on Power Systems, 2006, 21(4): 1883-1893.

[4] IEEE System Dynamic Performance Subcommittee. Voltage Stabi lity of Power System: Concepts. Analytical Tools, and Industry Experience, 90 Th0258-2-PWR, 1990.

[5] Wood A. J., and Wollenberg B. F., Power Generation Operation and Control. John Wiley \& Sons, Inc., 1996.

[6] Hur D., Park J. K., Kim B. H., and Son K. M., Security constrained optimal power flow for the evaluation of transmission capability on Korea electric power system. IEEE Power Engineering Society Summer Meeting, 2001: 1133-1138. 
[7] Bakirtzis A. G., Biskas P. N., Zoumas C. E., and Petridis V., Optimal power flow by enhanced ganetic algorithm. IEEE Trans. on Power Systems, 2002, 17(2): 229-236.

[8] Yuryevich J., and Wong K. P., Evolutionary programming based optimal power flow algorithm. IEEE Trans. on Power Systems, 1999, 14(1): 1245-1250.

[9] Vlachogiannis J. G., Haziargyriou N. D., and Lee K. Y., Ant colony system-based algorithm for constrained load flow problem. IEEE Trans. on Power Systems, 2005, 20(3): 1241-1249.

[10] Abido M. A., Optimal power flow using particle swarm optimization. Electrical Power and Energy Systems, 2002, 24(1): 563571.

[11] Lin W. M., Su Y. S., Chin H. C., and Teng J. H., Three-phase unbalanced distribution power flow solutions with minimum data preparation. IEEE Transactions on power Systems, 1999, 143(1): 1178-1183.

[12] Lin W. M., Huang C. H., and Zhan T. S., A hybrid current-power optimal power flow technique. IEEE Trans. on Power Systems, 2008, 23(1): 177-185.

[13] Huang S. J., An immune-based optimization method to capacitor placement in a radial distribution system. IEEE Trans. on Power Delivery, 2000, 15(2): 744-749. 